\title{
Hyperspectral Camera System
}

National Cancer Institute

\section{Source}

National Cancer Institute. Hyperspectral Camera System. NCI Thesaurus. Code C160679.

A proprietary imaging system that combines three sensors for spectral imaging. For 2D reference, and to facilitate annotating the data, a high resolution (full HD) color image, recorded with an RGB-sensor, is included with each hyperspectral dataset. 